\title{
The collagenolytic action of MMP-1 is regulated by the interaction between the catalytic domain and the hinge region
}

\author{
Giovanni Francesco Fasciglione $\cdot$ Magda Gioia $\cdot$ Hiroki Tsukada \\ Jian Liang • Riccardo Iundusi · Umberto Tarantino • \\ Massimo Coletta $\cdot$ Tayebeh Pourmotabbed $\cdot$ Stefano Marini
}

Received: 14 November 2011 / Accepted: 26 February 2012

(C) SBIC 2012

\begin{abstract}
The role of the hinge region in the unwinding and cleavage of type I collagen by interstitial collagenase (MMP-1) has been studied at $37{ }^{\circ} \mathrm{C}$ and $\mathrm{pH}$ 7.3. The collagenolytic processing by MMP-1 displays a very similar overall rate for both chains of collagen I, even though the affinity is higher for the $\alpha-1$ chain and the cleavage rate is faster for the $\alpha-2$ chain. MMP-1 binding to collagen I brings about a significant unwinding of the triple-helical arrangement only after the first cleavage step of the $\alpha-1$ and $\alpha-2$ chains. The proteolytic processing by wild-type MMP1 on a synthetic substrate and collagen I has been compared with that observed for site-directed mutants obtained either by truncating the hinge region $(\Delta 255-272)$ or by individually replacing the conserved amino acids Val268, Gly272, and Lys277 of the hinge region with residues observed for the corresponding position in stromelysin-1 (MMP-3), a noncollagenolytic metalloproteinase. The $\Delta 256-272$ mutant has no collagenolytic activity, clearly demonstrating the crucial role of this region for the enzymatic
\end{abstract}

G. F. Fasciglione - M. Gioia - R. Iundusi - U. Tarantino ·

M. Coletta $\cdot$ S. Marini $(\varangle)$

Department of Clinical Sciences and Translational Medicine,

University of Roma Tor Vergata,

Via Montpellier 1, 00133 Rome, Italy

e-mail: stefano.marini@uniroma2.it

M. Gioia $\cdot$ M. Coletta $\cdot$ S. Marini

Interuniversity Consortium for the Research on the Chemistry

of Metals in Biological Systems (CIRCMSB),

Via C. Ulpiani 27, 70125 Bari, Italy

H. Tsukada $\cdot$ J. Liang · T. Pourmotabbed

Department of Microbiology,

Immunology and Biochemistry,

University of Tennessee Health Science Center,

Memphis, TN 38163, USA processing of collagen I. However, among various mutants investigated, only Gly272Asp shows a dramatically reduced enzymatic activity both on the synthetic substrate and on collagen I. This effect, however, is clearly related to the substituting residue, since substitution of Ala or Asn for Gly272 does not have any effect on the kinetic properties of MMP-1. These data suggest that the substrate specificity of MMP-1 is dictated by the reciprocal structural relationships between the catalytic domain and the carboxyterminal domain through the conformational arrangement of the hinge region.

Keywords Fibroblast collagenase - Collagen I · Collagenolytic cleavage mechanism - Kinetics . Hinge region

\section{Introduction}

Matrix metalloproteinases (MMPs) are a family of $\mathrm{Zn}^{2+}$ dependent endopeptidases involved in the proteolytic processing of several components of the extracellular matrix, such as collagens, proteoglycans, and fibronectin [1-4]. They have been implicated in several physiological and pathological processes, such as skeletal growth and remodeling, wound healing, cancer, arthritis, and multiple sclerosis [5-10]. The MMP domain usually consists of an amino-terminal prodomain (which is removed upon activation of the enzyme), a catalytic domain (where the active $\mathrm{Zn}^{2+}$ is located), and a carboxy-terminal hemopexin-like domain, which is separated from the catalytic domain by a Pro-rich linker peptide (also called the hinge region) of variable length [1]. The MMP class can be classified into five main groups, namely, (1) collagenases (i.e., MMP-1, MMP-8, and MMP-13), which are able to cleave fibrillar 
collagen and bind collagen preferentially through the hemopexin-like domain [11-13], (2) gelatinases (i.e., MMP-2 and MMP-9), which are characterized by an additional domain, called collagen binding domain, that is made of three fibronectin II like repeats located in their catalytic domain and represents the preferential binding domain for fibrillar collagen I [14], (3) stromelysins (i.e., MMP-3, MMP-10 and MMP-11), which are unable to cleave fibrillar collagen I, (4) matrilysins (i.e., MMP-7 and MMP-26), which lack the hemopexin-like domain and are able to process collagen IV but not collagen I [15], and (5) membrane-bound MMPs (i.e., MMP-14, MMP-15, MMP-16 and MMP-17), whose carboxy-terminal domain contains an intermembrane region (or segment), followed by a short cytoplasmic tail. MMP-14 is the only membranebound MMP which has been shown to be able to cleave fibrillar collagen I [16].

Interstitial collagens I, II, and III are triple-helical proteins and they represent the main structural components of many connective tissues, such as cartilage, bone, skin, tendons, and ligaments [17]. Triple-helical collagen is a rodlike protein, whose self-association forms fibrils, which represent the scaffold of the tissue architecture in animals [18]. These abundant proteins are structurally unique molecules consisting of three $\alpha$ chains; two types of lefthanded $\alpha$ chains can coil into each other, forming a righthanded triple helix. This consists largely of the repeated sequence Gly-X-Y, where X and Y are mainly Pro and 4-trans-L-hydroxyproline residues, respectively. These types of sequences favor a left-handed poly-Pro II helix conformation, and the interwinding of the three chains (constituting the supersecondary structure of the collagenous triple helix) is stabilized through hydrogen bonds nearly perpendicular to triple-helical axis [19]. The triple helix is made of two $\alpha-1$ chains and one $\alpha-2$ chain, and this structural assembly is characterized by a typical dichroic signal, peaking at $224 \mathrm{~nm}$ [20], which decreases only upon gross unwinding. Since physiological collagenolysis is crucial for several biological processes, such as tissue repair and remodeling, angiogenesis, and wound healing $[3,21]$, and collagenases are the main MMPs involved in collagen turnover and remodeling [22-24], a clarification of the mechanism of processing of the triple helix by collagenases is of the utmost importance. Structural data on fibrillar collagen I [25-27] indeed have shown that, on the basis of the available three-dimensional structures of collagenases [28, 29], the triple-helical structural assembly does not allow collagen I to come into close contact with the catalytic site of collagenases. Therefore, a more complex mechanism of substrate recognition must be postulated, where an exosite topologically distinct from the active site interacts with the substrate (likely through the hemopexin-like domain in collagenases; see [14]) with a very limited alteration of the triple-helical arrangement of collagen I [30-32]. This first step is then followed by the proteolytic cleavage, performed by the active site. This process requires an induced-fit conformational change, which allows the active site to come into contact with the primary cleavage site [33]. In this respect, it has recently been outlined that various MMPs display drastically different flexibility between the two main structural domains (i.e., the hemopexin-like and the catalytic domains), envisaging that this feature may be important for the functional modulation [34, 35]. However, despite the fact that site-directed mutagenesis studies suggest that the linker hinge region plays a role in collagenolysis in MMP-1 $[12,36,37]$, the initial alteration in the triple-helical collagen molecule upon MMP-1 binding and its modulation remains elusive, even though some recent information has started to be collected [38].

We have performed a quantitative comparison of the collagenolytic activities of hinge-region mutants of MMP1 in an attempt to clarify in better detail the modulatory role of the linker region. We have found that a site-directed mutation, which makes the central portion of the linker hinge region rigid, drastically alters the enzymatic activity of MMP-1, mostly affecting the catalytic rate constant and abolishes substrate specificity of the enzyme toward $\alpha-1$ and $\alpha-2$ chains of collagen I. This suggests an important role for this region in communicating conformational changes between the carboxyl-terminal hemopexin domain and the catalytic domain.

\section{Materials and methods}

Protein preparation and purification

Recombinant wild-type MMP-1 and site-directed mutants Val268Pro, Gly272Glu, and Lys277Ala were produced as previously described $[37,39]$. The construction of $\mathrm{pET} / \mathrm{FC}$ containing the full-length complementary DNA (cDNA) for MMP-1 has been described previously [39].

The deletion $(\Delta 255-272)$ and the site-directed Gly272Ala and Gly272Asn mutations were introduced in MMP-1 by PCR using pET/FC as the template. The $5^{\prime}$ mutagenic primers $5^{\prime}$-TGTTCAGCTAGCTCAGGATGAC ATTGAT $\triangle$ GGCCCACAAACCCCA-3', 5'-ATATGGAC GTTCCCAAAATCCTGTCCAGCCCATCGCCCCACAA ACC- $3^{\prime}$ and $5^{\prime}$-ATATGGACGTTCCCAAAATCCTGTCC AGCCCATCAACCCACAAACC- $3^{\prime}$ were used to independently delete amino acid residues 255-272 from the hinge region and substitute either Ala or Asn, respectively, for Gly272. The $\Delta 255-272$ mutagenic primer contains cDNA sequences that code for the amino acids flanking amino acid residues 255 and 272 (the corresponding 
nucleotides are underlined), causing the deletion of the 255-272 peptide sequence from the hinge region. The $3^{\prime}$ primer, 5'-TTTGGACTCACACCATGTGTTTTCCATTC AAATTAG- ${ }^{\prime}$, that was used to construct all of the abovementioned mutations was complementary to the $3^{\prime}$ untranslated region of the cDNA and contained a unique DraIII restriction site.

The PCR product corresponding to the $\Delta 255-272$ deletion mutant product was digested with NheI and DraIII, and the resulting DNA fragment was ligated with NheI/DraIII DNA fragment of $\mathrm{pET} / \mathrm{FC}$ to produce expression vector $\mathrm{pET} / \mathrm{FC} / \Delta 255-272$. The resulting expression vector coded for an MMP-1 mutant enzyme in which the 255-272 peptide fragment was deleted. This was confirmed by dideoxy sequencing, which verified the presence of the correct cDNA junction coding for the following amino acid sequence:

$$
\ldots{ }^{254} \mathrm{I}^{255} \mathrm{D}-{ }^{272} \mathrm{G}^{273} \mathrm{P} \ldots
$$

The PCR products corresponding to Gly272Ala and Gly272Asn mutations were digested with MaeIII and DraIII restriction enzymes and ligated with SphI/DraIII and SphI/MaeIII fragments from $\mathrm{pET} / \mathrm{FC}$ to produce expression vectors $\mathrm{pET} / \mathrm{FC} / \mathrm{G} 272 \mathrm{~A}$ and $\mathrm{pET} / \mathrm{FC} / \mathrm{G} 272 \mathrm{~N}$, respectively. All of the PCR-derived DNAs for these constructs were sequenced to confirm that the desired mutations were the only mutations generated by the PCR. Plasmids encoding the mutant enzymes were introduced into Escherichia coli BL21(DE3) cells and were maintained in $50 \mu \mathrm{g} / \mathrm{ml}$ carbenicillin.

Batimastat (BB-94), a peptidomimetic synthetic inhibitor which stoichiometrically inhibits MMPs, was kindly provided by British Biotech Pharmaceuticals (Oxford, UK).

\section{Preparation of type I collagen}

Soluble collagen I was prepared by standard ammonium sulfate precipitation procedures from tail tendons of 8-week-old Wistar rats, as previously described [40]. The amount of collagen was quantified as described by Bradford [41].

Since purification of type I collagen often results in contamination by type III collagen, the purity of the final collagen I solution was confirmed by running a degradation experiment with wild-type MMP-1. In the type I collagen purification, no type III collagen degradation products were observed.

To rule out any collagenase contamination of our collagen I preparation, we incubated solutions of type I collagen with 4-aminophenylmercuric acetate so as to activate any latent enzyme. Sodium dodecyl sulfate polyacrylamide gel electrophoresis (SDS-PAGE) of these solutions, after 6 days of incubation, did not indicate any degradation, clearly ruling out any contamination by external collagenases.

Further, the native triple-helical structure of the collagen I preparation was tested by incubating the purified collagen solution with trypsin in an enzyme-to-substrate ratio of 1:10 ratio for $3 \mathrm{~h}$ at $28^{\circ} \mathrm{C}$; no appreciable degradation was observed. The triple-helical stability of collagen I was also followed by circular dichroism (CD); as already reported [32], gel-entrapped collagen samples of each collagen purification were monitored by $\mathrm{CD}$ for as long as 3 days at $37{ }^{\circ} \mathrm{C}$ and no significant change in the $\mathrm{CD}$ spectra was detected.

Lyophilized collagen I was stored at $-80{ }^{\circ} \mathrm{C}$, and stock solutions were prepared as needed. No significant difference in the triple-helical content and in the stability of collagen I was detected among different preparations of collagen I after the solubilization.

Determination of kinetic and thermodynamic parameters

The collagen I solutions (between 0.2 and $1.4 \mu \mathrm{M}$ ) were incubated with either active wild-type MMP-1 or different mutant enzymes (at concentrations between 0.1 and $2 \mathrm{nM}$ ) at $37{ }^{\circ} \mathrm{C}$ in $0.05 \mathrm{M}$ tris(hydroxymethyl)aminomethane (Tris)/ $\mathrm{HCl}, \mathrm{pH} 7.3$ buffer containing $0.1 \mathrm{M} \mathrm{NaCl}$ and $0.01 \mathrm{M} \mathrm{CaCl}_{2}$. Small aliquots were harvested at different time intervals, the reactions were stopped by the addition of SDS-PAGE loading buffer containing $20 \mathrm{mM}$ EDTA, and the samples were kept frozen at $-80{ }^{\circ} \mathrm{C}$ until use. The reaction products were separated on $7.5 \%$ SDS-PAGE gels [42] and visualized by either the Coomassie blue or the silver staining method. Broad-spectrum protein markers (Bio-Rad, Hercules, CA, USA) were used as molecular weight standards. The intensity of collagen I fragments at different time intervals was determined by image analysis software (Image Quant TL, Amersham Biosciences, UK); spots were converted to their corresponding protein concentrations using standard substrate solutions. For each $\alpha$ chain (i.e., $\alpha-1$ and $\alpha-2$ chains), the substrate disappearance rates were derived at each collagen concentration employed. The initial velocities were measured for different time intervals, corresponding to the degradation of less than $10 \%$ of the substrate, but always for less than $1 \mathrm{~h}$, that is, the time period during which the rate remained constant. This ensured a steady-state condition for the first cleavage step, and this was a prerequisite for the subsequent analysis step, which was based on the observation of an inverse linear correlation between velocity and substrate concentration according to the Lineweaver-Burk equation (Eq. 1) and Eadie-Hofstee linear regression (Eq. 2): 
$\frac{E_{0}}{v}=\frac{K_{\mathrm{m}}}{k_{\mathrm{cat}}} \cdot \frac{1}{[\mathrm{~S}]}+\frac{1}{k_{\mathrm{cat}}}$

$\frac{v}{E_{0}}=k_{\text {cat }}-\frac{v}{E_{0}[\mathrm{~S}]} K_{\mathrm{m}}$,

where $E_{0}$ is the total enzyme concentration, $v$ is the actual rate $\left(\mathrm{mol} \mathrm{s}^{-1}\right), K_{\mathrm{m}}$ is the Michaelis-Menten equilibrium constant (mol), $k_{\text {cat }}$ is the rate-limiting-step kinetic constant $\left(\mathrm{s}^{-1}\right)$ and $[\mathrm{S}]$ is the substrate concentration. Linear regression plots were constructed from the velocity data, and the catalytic parameters $k_{\mathrm{cat}}$ and $K_{\mathrm{m}}$ were extracted.

\section{Circular dichroism experiments}

CD spectra were recorded with a JASCO J710 spectropolarimeter equipped with a thermostatted cell holder and connected to a data station for signal averaging and processing. All spectra are averages of six scans and were recorded employing quartz cells of 2-mm path length.

For the spectroscopic observations, collagen I was entrapped in agarose gel by mixing the solubilized protein with a $1 \%$ low melting point agarose solution at $37{ }^{\circ} \mathrm{C}$ and $\mathrm{pH}$ 3.0. After rapid stirring, the mixture was poured on a simple gel casting (Mini-PROTEAN II, Bio-Rad) and immediately cooled to obtain the gel. The final concentrations were $0.5 \%$ for agarose and $1 \mu \mathrm{M}$ for collagen. The thickness of the homogeneous gel was $1 \mathrm{~mm}$. As a control, CD measurements were performed on samples of agarose gel, which did not show any ellipticity, and the data obtained were considered as the background. In addition, no spectral differences were observed between 200 and $250 \mathrm{~nm}$ (a spectral region representative of the triplehelical arrangement of collagen I; see [43]) for the CD spectrum obtained from gel-entrapped collagen at $\mathrm{pH} 3.0$ with respect to that obtained from solubilized collagen I at the same $\mathrm{pH}$ and concentration conditions (data not shown); this indicates that the collagen conformation was not altered by the gel-entrapment procedure.

For the kinetic experiments, slices of suitable size were cut off from the homogeneous gel and kept overnight in buffer solution to reach the desired $\mathrm{pH}$ without the formation of aggregates and were then used for CD measurements [28]. The CD spectra obtained for the collagen fragments are in good agreement with those obtained for collagen-like peptides, showing the characteristic triple helix maximum in the 223-225-nm range [44]. Therefore, we achieve a "quasi-native" model for evaluating structural arrangements during proteolytic processes on type I collagen [28].

The kinetics of the unwinding of the triple helix was obtained in $0.05 \mathrm{M}$ Tris/ $\mathrm{HCl}, 0.1 \mathrm{M} \mathrm{NaCl}, 0.01 \mathrm{M} \mathrm{CaCl}_{2}$ solution at $37{ }^{\circ} \mathrm{C}$ and $\mathrm{pH} 7.3$ by recording all the spectra over the range from 200 to $250 \mathrm{~nm}$ as a function of the time of incubation with different MMP-1 forms. The collagen and enzyme concentrations used in these experiments were the same as those in the fragmentation kinetics experiments described earlier. The variation of the molar ellipticity at $224 \mathrm{~nm}$ as a function of time allowed us to obtain values for the time evolution of the unwinding process. The conformational changes of the triple-helical arrangement upon binding of MMP-1 were checked by incubating agarose-gel-entrapped collagen I ( $1 \mu \mathrm{M}$ concentration) with $5 \mu \mathrm{M}$ MMP-1 fully inhibited with a molar excess of batimastat.

Determination of kinetic parameters with a synthetic substrate

Enzymatic activity was measured by employing a fluorogenic substrate, namely, MCA-Pro-cyclohexylalanine-Glynor-Val-His-Ala-DPA-NH $\mathrm{NH}_{2}$, where MCA is (7-methoxycoumarin-4-yl)acetyl and DPA is $N$-3-(2,4-dinitrophenyl)L-2,3-diaminopropionyl (MCA-1; Calbiochem, La Jolla, CA, USA). Experiments were conducted at a final $0.01 \mathrm{nM}$ concentration of purified activated wild-type MMP-1 or site-directed mutants of MMP-1 (namely, Gly272Asp, Gly272Asn, and hinge-deleted MMP-1) at $37{ }^{\circ} \mathrm{C}$ in $0.05 \mathrm{M}$ Tris/HCl, $0.1 \mathrm{M} \mathrm{NaCl}, 0.01 \mathrm{M} \mathrm{CaCl}_{2}$ plus $0.05 \%$ Brij 35 buffered at $\mathrm{pH} 7.3$, and with different concentrations of MCA-1 (from 0.75 to $3 \mu \mathrm{M}$ ).

Enzymatic processing of the fluorogenic synthetic substrate was followed by spectrofluorimetry (Eclipse, Varian), employing $325 \mathrm{~nm}$ as the excitation wavelength and following the fluorescence at $393 \mathrm{~nm}$. Data were analyzed according to Eqs. 1 and 2.

\section{Results}

\section{Wild-type MMP-1}

Figure 1 shows the SDS-PAGE of collagen I exposed to wild-type MMP-1 for different time intervals at $37{ }^{\circ} \mathrm{C}$ and at $\mathrm{pH}$ 7.3. It is evident that the two chains are degraded progressively, bringing about the formation of fragments of lower molecular weight (corresponding to the $1 / 4$ and $3 / 4$ fragments; see [45]). This first cleavage step displayed Michaelis-Menten behavior, as indicated by the inverse linear relationships between the velocity of the process and substrate concentration (data not shown). Catalytic parameters are reported in Table 1 for the synthetic substrate as well as for the two collagen chains. As shown in this table, the overall enzymatic activity (i.e., $k_{\mathrm{cat}} / K_{\mathrm{m}}$ ) of MMP-1 is about tenfold higher for the synthetic substrate than for both collagen chains, and this is mostly due to a higher affinity of the enzyme for the synthetic substrate 


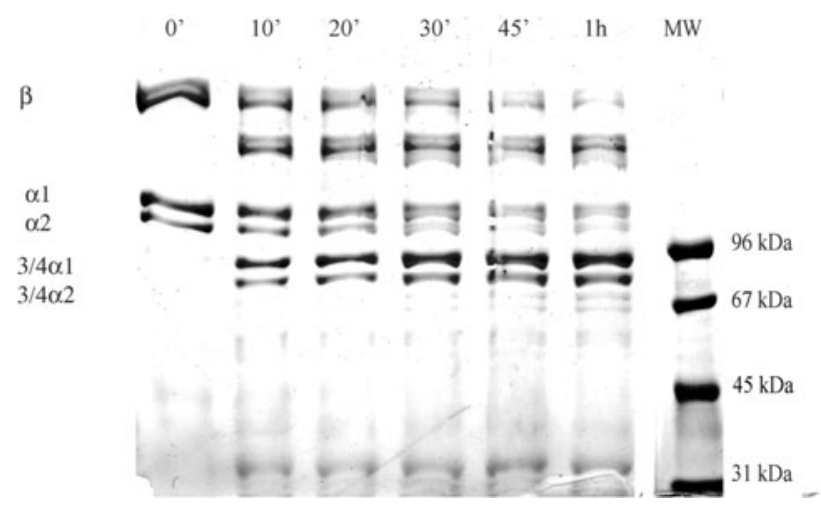

Fig. 1 Sodium dodecyl sulfate polyacrylamide gel electrophoresis (SDS-PAGE) of the kinetics of fragmentation of collagen I by $18 \mathrm{nM}$ wild-type MMP-1 at $37{ }^{\circ} \mathrm{C}$ and $\mathrm{pH}$ 7.3. Left column collagen I sample in the absence of enzyme, right column molecular weight markers, intermediate columns samples of collagen I incubated with wild type MMP-1 for the intervals indicated. For further details, see the text. $M W$ molecular mass

(i.e., a lower $K_{\mathrm{m}}$ ). Further, as shown in Table 1, MMP-1 cleaves the $\alpha-2$ chain seven to eight times faster than the $\alpha$ 1 chain, as demonstrated by the higher $k_{\text {cat }}$ value (3.8 vs. $0.55 \mathrm{~s}^{-1}$ ), whereas it has a much lower affinity for the $\alpha-2$ chain (the $K_{\mathrm{m}}$ values for the $\alpha-1$ and $\alpha-2$ chains are $4.3 \times 10^{-6} \mathrm{M}$ and $2.3 \times 10^{-5} \mathrm{M}$, respectively; see Table 1); the overall result is a similar proteolytic efficiency for the two chains (as from the values of $k_{\text {cat }} / K_{\mathrm{m}}$; see Table 1).

To obtain information on the temporal evolution of the unwinding of the triple helix, the enzymatic processing of collagen I by wild-type MMP-1 was also followed by CD.
Figure $2 \mathrm{a}$ reports the time course of the formation of the $3 / 4$ fragment for the $\alpha-1$ and $\alpha-2$ chains. In conjunction with the data obtained for the unwinding of the triple helix following the decrease of ellipticity at $224 \mathrm{~nm}$ [46, 47] (Fig. 2b), these data clearly demonstrate that during the first cleavage step (occurring within the first $2 \mathrm{~h}$; see Fig. 2a) no relevant unwinding of the triple helix occurs. Unwinding of collagen I occurs at longer incubation times together with the appearance of smaller fragments (see Fig. 2a). This finding suggests that binding of MMP-1 to the triple-helical collagen I does not grossly perturb the triple helix, which only extensively unwinds after the first cleavage step, when the $3 / 4$ fragment is further cleaved to lower molecular weight fragments (see Fig. 2a, b). This interpretation is supported by the observation that addition of a fully inhibited MMP-1 (after the addition of a molar excess of the inhibitor batimastat) does not alter the ellipticity of collagen I (Fig. 2c). Thus, since MMP-1 is known to interact with collagen I through its hemopexinlike domain [31], full inhibition of the catalytic activity has been demonstrated not to impair the interaction of MMP-1 with collagen I [19, 48, 49], and the lack of changes in ellipticity upon addition of fully inhibited MMP-1 clearly rules out any gross modification of the triple-helical structure following the interaction, as it occurs instead for MMP-2 [32]. However, since the whole triple helix is known to be too large to fit into the catalytic site of MMP-1 [27], this finding does not rule out the possibility that the interaction of MMP-1 with collagen might induce some small local perturbation in the triple-helical molecule to adapt the individual chain to the active site of MMP-1; it
Table 1 Catalytic parameters for the enzymatic processing of the synthetic substrate and two chains of collagen I at $37{ }^{\circ} \mathrm{C}$ and $\mathrm{pH} 7.3$ by wild-type MMP1 and site-directed mutants of MMP-1

\begin{tabular}{llll}
\hline & $k_{\text {cat }} / K_{\mathrm{m}}\left(\mathrm{M}^{-1} \mathrm{~s}^{-1}\right)$ & $k_{\text {cat }}\left(\mathrm{s}^{-1}\right)$ & $K_{\mathrm{m}}(\mathrm{M})$ \\
\hline$\alpha-1$ chain & & & \\
Wild-type MMP-1 & $1.3( \pm 0.2) \times 10^{5}$ & $0.55( \pm 0.07)$ & $4.3( \pm 0.6) \times 10^{-6}$ \\
Lys277Ala MMP-1 & $9.8( \pm 1.1) \times 10^{4}$ & $0.85( \pm 0.11)$ & $8.7( \pm 1.0) \times 10^{-6}$ \\
Gly272Ala MMP-1 & $1.9( \pm 0.3) \times 10^{5}$ & $1.10( \pm 0.16)$ & $5.8( \pm 0.7) \times 10^{-6}$ \\
Gly272Asp MMP-1 & $1.2( \pm 0.2) \times 10^{3}$ & $0.06( \pm 0.01)$ & $5.0( \pm 0.7) \times 10^{-5}$ \\
Gly272Asn MMP-1 & $1.5( \pm 0.2) \times 10^{5}$ & $0.51( \pm 0.07)$ & $3.5( \pm 0.5) \times 10^{-6}$ \\
Val268Pro MMP-1 & $1.1( \pm 0.2) \times 10^{5}$ & $0.57( \pm 0.06)$ & $5.2( \pm 0.7) \times 10^{-6}$ \\
$\alpha-2$ chain & & & \\
Wild-type MMP-1 & $1.6( \pm 0.2) \times 10^{5}$ & $3.80( \pm 0.48)$ & $2.3( \pm 0.4) \times 10^{-5}$ \\
Lys277Ala MMP-1 & $1.7( \pm 0.3) \times 10^{5}$ & $4.25( \pm 0.56)$ & $2.5( \pm 0.4) \times 10^{-5}$ \\
Gly272Ala MMP-1 & $2.4( \pm 0.4) \times 10^{5}$ & $9.10( \pm 1.23)$ & $3.8( \pm 0.5) \times 10^{-5}$ \\
Gly272Asp MMP-1 & $9.0( \pm 1.1) \times 10^{2}$ & $0.04( \pm 0.01)$ & $4.4( \pm 0.6) \times 10^{-5}$ \\
Gly272Asn MMP-1 & $3.4( \pm 0.5) \times 10^{5}$ & $12.20( \pm 1.87)$ & $3.6( \pm 0.5) \times 10^{-5}$ \\
Val268Pro MMP-1 & $1.5( \pm 0.3) \times 10^{5}$ & $4.02( \pm 0.52)$ & $2.7( \pm 0.4) \times 10^{-5}$ \\
Synthetic substrate & & & \\
Wild-type MMP-1 & $1.2( \pm 0.2) \times 10^{6}$ & $1.03( \pm 0.21)$ & $8.0( \pm 1.4) \times 10^{-7}$ \\
Gly272Asp MMP-1 & $5.0( \pm 0.7) \times 10^{5}$ & $0.14( \pm 0.03)$ & $2.7( \pm 0.4) \times 10^{-7}$ \\
Gly272Asn MMP-1 & $3.3( \pm 0.5) \times 10^{6}$ & $1.63( \pm 0.32)$ & $4.9( \pm 0.7) \times 10^{-7}$ \\
\hline
\end{tabular}



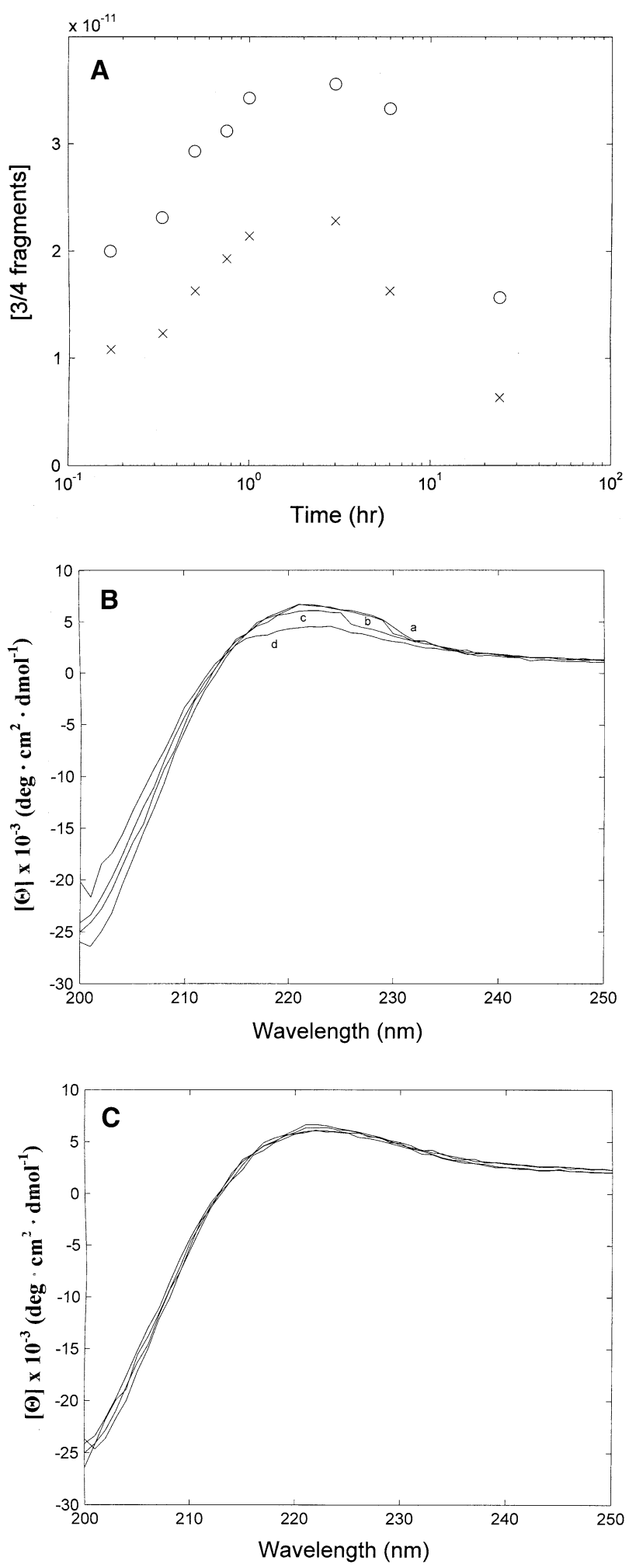

simply means that this perturbation may be topologically limited to the immediate surroundings of the binding site, as suggested by Chung et al. [31], without spreading over a significant portion of the collagen molecule. This is consistent with recent observations [50] that at room
Fig. 2 A Time evolution of the formation and disappearance of $3 / 4$ fragments from the $\alpha-1$ chain (circles) and the $\alpha$-2 chain (crosses) after incubation of $1.2 \mu \mathrm{M}$ collagen with $18 \mathrm{nM}$ wild-type MMP-1 at $37^{\circ} \mathrm{C}$ and $\mathrm{pH}$ 7.3. B Circular dichroism spectra of agarose-gelentrapped collagen I at $37{ }^{\circ} \mathrm{C}$ and $\mathrm{pH} 7.3$ before incubation with $18 \mathrm{nM}$ wild-type MMP-1 (spectrum a) and after $1 \mathrm{~h}$ (spectrum $b$ ), $7 \mathrm{~h}$ (spectrum c), and $24 \mathrm{~h}$ (spectrum d) of incubation with $18 \mathrm{nM}$ wildtype MMP-1. C Circular dichroism spectra of agarose-gel-entrapped $1 \mu \mathrm{M}$ collagen before and after addition of $5 \mu \mathrm{M}$ wild-type MMP-1 fully inhibited by a molar excess of batimastat. For further details, see the text

temperature collagen adopts a partially unfolded conformation that is complementary to the active sites of MMP-1 and MMP-8, suggesting that an enzyme-mediated unwinding of the collagen molecule is not required for collagenolysis.

\section{Site-directed mutants of MMP-1}

Using site-directed mutagenesis experiments, we have previously shown that the hinge region plays a role in the catalytic activity of MMP-1 [11,33]. To assess the role of the hinge region in collagen presentation to and proteolytic processing by the active site, we replaced the conserved amino acid residues Val268, Gly272, and Lys277 in this region of the MMP-1 molecule with residues (namely, Pro, Glu, and Ala, respectively) which are present in the noncollagenolyic MMP stromelysin [37, 51]. As shown in Fig. 3, Val 268, Gly272, and Lys277 are located at three different positions along the hinge region: Val268 is close to the catalytic domain of MMP-1, whereas Gly272 and Lys 277 are located approximately in the middle and close to the hemopexin-like domain, respectively.

As reported in Table 1, the catalytic parameters (i.e., $k_{\text {cat }}$ and $K_{\mathrm{m}}$ ) of the Val268Pro mutant for both $\alpha-1$ and $\alpha-2$ chains are very similar to those observed for wild-type

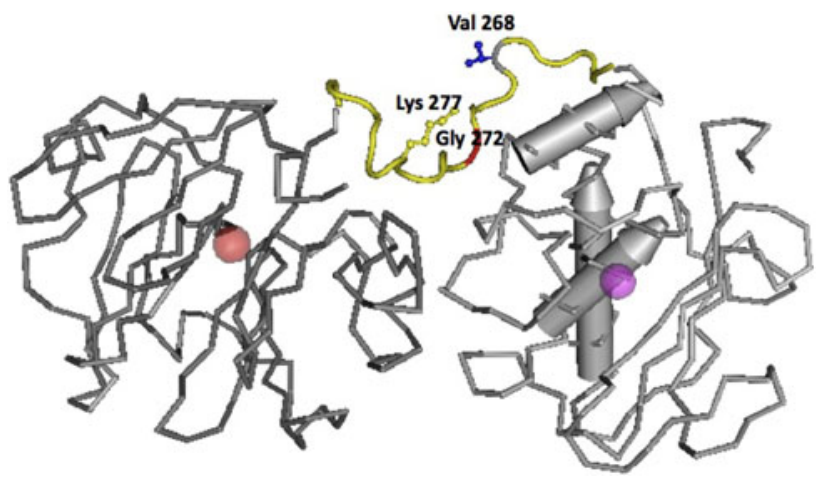

Fig. 3 Ribbon diagram representation of the full-length MMP-1 structure. This figure was modeled after the X-ray structure of porcine synovial collagenase [29]. Mutated amino acids Val268, Gly272, and Lys277 of the hinge region are shown. The figure was obtained using $\mathrm{Cn} 3 \mathrm{D}$, version 4.1 (National Center for Biotechnology Information) 
MMP-1, clearly indicating that this mutation leaves the MMP-1 activity unchanged. In the case of the Lys277Ala mutant, the overall enzymatic activity toward the $\alpha-1$ chains is slightly decreased with respect to wild-type MMP-1. The mutation has slightly increased both the $k_{\text {cat }}$ and $K_{\mathrm{m}}$ values of the enzyme for the $\alpha$-1-chain, resulting in an overall $25 \%$ decrease in catalytic efficiency of the enzyme toward the collagen $\alpha$-1-chain. This mutation, however, does not affect the processing of the $\alpha-2$ chain (see Table 1).

Site-directed mutants of MMP-1 on the Gly272 residue

A completely different result was obtained when we replaced Gly272 with Asp, its corresponding amino acid residue in MMP-3. The catalytic efficiencies of the Gly272Asp mutant toward the $\alpha-1$ and $\alpha-2$ chains of type I collagen were 100- and 177-fold lower than those of wildtype MMP-1, respectively (Fig. 4, Table 1). Further, the Gly272Asp mutant was found to have a very similar catalytic efficiency toward the $\alpha-1\left(1.2 \times 10^{3} \mathrm{M}^{-1} \mathrm{~s}^{-1}\right)$ and $\alpha-2\left(9.0 \times 10^{2} \mathrm{M}^{-1} \mathrm{~s}^{-1}\right)$ chains of type I collagen. The observed low catalytic efficiency of the mutant enzyme toward the $\alpha-1$ chain was due to a decrease in both its catalytic activity toward the substrate (i.e., $k_{\text {cat }}$ ) and its affinity for the substrate (i.e., $K_{\mathrm{m}}$ ), whereas the low catalytic efficiency of the enzyme toward the $\alpha-2$ chain was attributed mainly to its low $k_{\text {cat }}$ value (Table 1 ). The fact that Gly272Asp cannot discriminate between $\alpha-1$ and $\alpha-2$ chains and that it has a significantly lower affinity for the $\alpha$ 1 chain as compared with the wild-type enzyme indeed suggests that Gly272 plays a dual role in MMP-1 activity, namely, (1) it is involved in the conformation of the catalytic site (thus altering the substrate cleavage velocity)

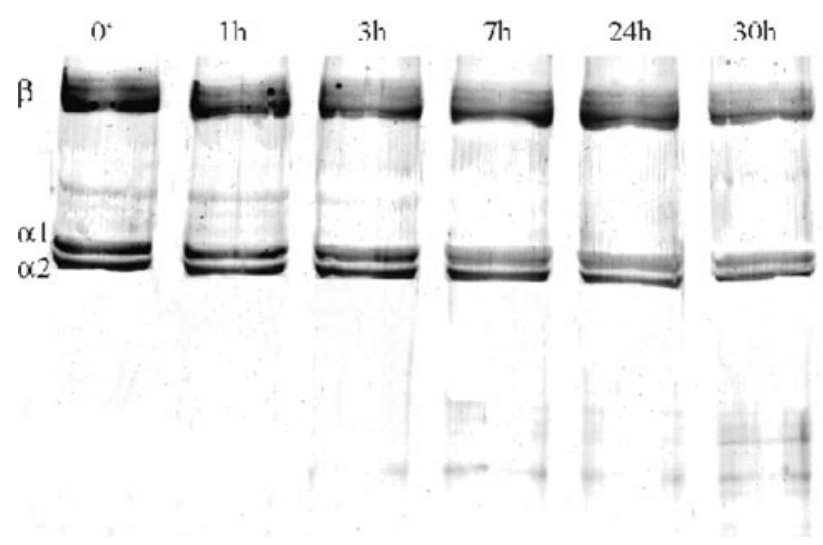

Fig. 4 SDS-PAGE electrophoresis of the kinetics of fragmentation of collagen I by $0.09 \mathrm{nM}$ Gly272Asp mutant of MMP- 1 at $37^{\circ} \mathrm{C}$ and $\mathrm{pH}$ 7.3. Left column collagen I sample in the absence of enzyme, other columns samples of collagen I incubated with Gly272Asp mutant of MMP-1 for the intervals indicated. For further details, see the text and (2) it modulates the interaction of the enzyme with the macromolecular substrate (thus affecting the MichaelisMenten constant $K_{\mathrm{m}}$ ), being particularly important for the binding of the $\alpha-1$ chain. This was also confirmed by the observation that the Gly272Asp mutant has three times higher affinity and seven times lower catalytic activity toward the synthetic substrate as compared with the wildtype enzyme (Table 1).

To further evaluate the role of Gly272 in type I collagenolysis of MMP-1, we replaced this residue with two other residues, namely, the nonpolar amino acid Ala and the polar uncharged amino acid Gln. In the case of the Gly272Asn mutant, all catalytic parameters with regard to the $\alpha-1$ chain are very similar to those of the wild-type enzyme, whereas for the $\alpha-2$ chain we observed only a threefold increase for the catalytic rate-limiting step (i.e., $\left.k_{\text {cat }}\right)$. Interestingly, the enzyme had higher affinity and higher catalytic activity toward the synthetic substrate with respect to the wild-type enzyme, indicating that the observed increase in catalytic activity of MMP-1 upon mutation is independent of the type of substrate. In the case of the Gly272Ala mutant, only a modest twofold increase of the $k_{\text {cat }}$ value for both collagen chains was observed (Table 1). Most importantly, unlike Gly272Asp, both Gly272Asn and Gly272Ala distinguish the $\alpha-1$ chain from the $\alpha-2$ chain, albeit with different catalytic activity and affinity compared with the wild type. Thus, as demonstrated in Table 1, the Gly272Asp mutant is the only enzyme mutant that shows very similar catalytic parameters for both the $\alpha-1$ chain and the $\alpha-2$ chain of type I collagen. Wild-type MMP-1 and all other site-directed mutants have fourfold to tenfold lower affinity and fivefold to 20 -fold higher catalytic activity toward the $\alpha-2$ chain than the $\alpha-1$ chain as demonstrated by their $k_{\text {cat }}$ and $K_{\mathrm{m}}$ values (Table 1). This result clearly indicates that in the Gly272Asp mutant the dramatic decrease of the rate constant for the catalytic step also affects the discrimination mechanism for the interaction with the two chains. These data suggest that the negative charge on this crucial residue of the hinge region disrupts the preferential interaction of MMP-1 with the $\alpha-1$ chain by the wild-type species (as demonstrated by the $K_{\mathrm{m}}$ values; Table 1 ).

\section{Hinge-deleted mutant $(\Delta 255-272 \mathrm{MMP}-1)$}

To further assess the role of hinge region in the activity of MMP-1, we partially deleted this region and determined the effect of mutation on the catalytic activity of the enzyme. Unlike the site-directed mutant enzymes, the $\Delta 255-272$ deletion mutant does not show any proteolytic activity toward synthetic substrates or collagen molecules, indicating that the lack of the hinge domain dramatically affects not only the macromolecular substrate recognition, 
but also the intrinsic catalytic activity of MMP-1. The total lack of enzymatic activity of the mutant enzyme could be due to a steric hindrance effect generated by the close proximity of the hemopexin-like carboxy-terminal domain to the active site.

\section{Discussion}

Excessive degradation of type I collagen is associated with a variety of human diseases, such as arthritis, tumor metastasis, and atherosclerosis [22]. Collagenases (such as MMP-1, MMP-8, and MMP-13) are able to cleave fibrillar collagen I, leading to the formation of two fragments corresponding approximately to $3 / 4$ and $1 / 4$ fragments of the mature collagen molecule $[31,45]$. CD data (see Fig. 2c) strongly indicate that collagenolysis by MMP-1 occurs without any gross alteration of the triple-helical structure of collagen I, like for the MMP-8 reaction [30, 32], and unwinding of the collagen molecule is not required for collagenolysis, as recently suggested also by others [38, 50]. Since the triple-helical collagen in the native conformation is too big to be accommodated by the active site of MMP-1, this observation raises the question of how the first step of collagenolysis occurs without gross unwinding of the triple helix.

We have presented a strong indication that the efficacy of MMP-1 in cleaving triple-helical collagen I stems from the capability of its active site to adopt a conformation that mediates collagen binding and catalysis. In this respect, important information comes from previous observations [11, 13], which pointed out the crucial role played by the hemopexin-like domain in facilitating the correct interaction at $37^{\circ} \mathrm{C}$ between the collagenases and collagen I. Furthermore, the functional relevance of the linker region is demonstrated by the observation that variations of amino acids in this hinge region indeed bring about alterations of the collagenolytic activity in both MMP-1 [12] and MMP-8 [36]. It is reasonable then to postulate a concerted action between the binding of collagen I by the hemopexin-like domain and the action of the catalytic site [52], where the intermediate hinge region indeed might play the role of a transmission gear between the two domains [37].

The binding of collagen I by the hemopexin-like domain apparently triggers a conformational change where the topological relationships between the catalytic domain and the hemopexin-like domain change [29]. This transition is mediated by the hinge region, exploiting its flexibility [35], which permits the concerted action between the binding of collagen I by the hemopexin-like domain and the catalytic site (Fig. 5a), thus reducing the energetic barrier for distortions of the single collagen helix and for catalysis (Fig. 3). This functional link should be related to the
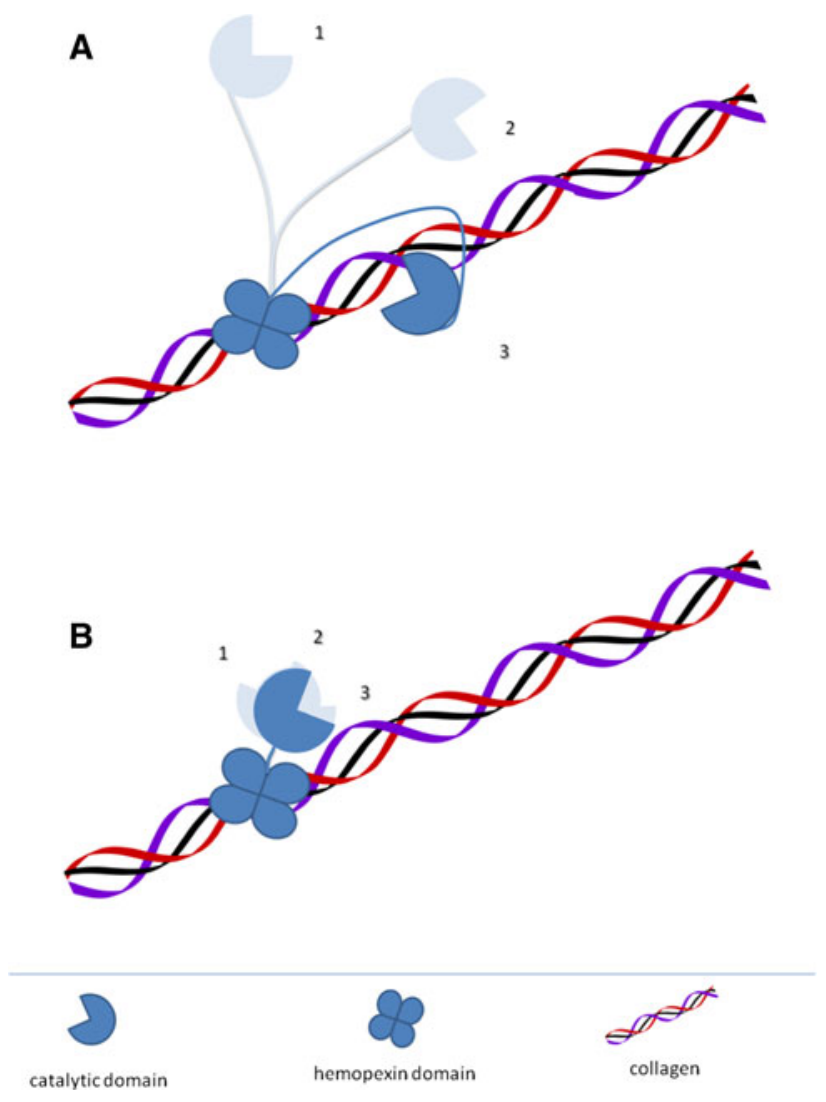

Fig. 5 Comparison of the different steps of interaction and cleavage of collagen by wild-type MMP-1 and a hinge-deleted mutant of MMP-1. A Wild-type MMP-1 is shown with the four-bladed hemopexin-like domain bound to the triple-helical collagen and the catalytic domain which binds the collagen molecule thanks to the flexibility of the linking hinge sequence. B The binding of collagen I by the hinge-deleted mutant of MMP-1 does not allow the catalytic domain to come into close contact with the collagen I triple helix because of the insufficiently flexible (or too short) hinge domain

occurrence of several hydrogen bonds between residues of the hinge region and those of both the catalytic and the hemopexin-like domains $[29,52]$. This crucial role for the hinge region as a transmission gear seems also to emerge from previous observations on MMP-8 [36], where Alascanning mutagenesis of the hinge region indeed brought about a dramatic decrease of the collagenolytic activity. However, within a general modulatory role played by this region, specific residues play more crucial roles, as demonstrated for Gly272 in MMP-1 (see Table 1) [37]. Substitution of Asp (the corresponding residue in noncollagenolytic MMPs, i.e., transin/stromelysin) for this residue substantially decreased the catalytic activity of the enzyme and abolished the specificity of the enzyme toward the $\alpha-1$ and $\alpha-2$ chains of collagen. Residue Gly 272 should provide the flexibility necessary for the active site of the enzyme to change conformation upon binding collagen, thus reducing the energy barrier for catalysis [37]. This relevant role also has a structural basis in that this linker region displays a 
poly-Pro II sequence (i.e., Pro-X-X-Pro), which has previously been postulated to help the hinge region assume an $\alpha$-chain-like structure and to stack into the triple-helical collagen [53]. This effect was not observed for the replacement of other residues of the hinge region by their corresponding residues in stromelysins. Thus, replacement of the positively charged residue, Lys277, in MMP-1 by its corresponding residue in transin/stromelysin, Ala, had virtually no effect on the collagenolytic activity of the enzyme, and the same was true for the substitution of Pro for Val268 (Table 1). These results clearly indicate that the low catalytic activity and the lack of discrimination between the $\alpha-1$ and $\alpha-2$ chains of collagen in the Gly272Asp mutant are not simply related to an unbalancing between positive and negative charges of the hinge region and/or an alteration of its bending capability, but other factors might come into play. In this respect, it is very important to remark that also for a synthetic substrate, whose interaction with MMP-1 should not involve the hemopexin-like domain, the Gly272Asp mutation brings about a tenfold decrease of the catalytic rate-limiting step $k_{\text {cat }}$ (Table 1 ), that is, to an extent very similar to what is observed with both chains of collagen (Table 1). This feature indeed suggests that mutation at this residue of the hinge induces a conformational change in the active site so as to reduce the cleavage rate constant with both small synthetic and large macromolecular substrates. However, this effect must be mostly attributed to the negative charge introduced by the mutation in Gly272Asp, since replacement of this residue with several uncharged amino acids as in Gly272Ala and Gly272Asn did not have a significant effect on the catalytic parameters of the enzyme (Table 1). Thus, since this rate is likely controlled by the movement of the catalytic domain onto the cleavage site once the hemopexin-like domain has bound the substrate, the hinge region (and in particular Gly272 as a bending center) comes into play by regulating the energy barrier for this movement (Fig. 5b). A somewhat different mechanism should operate for the collagenolysis of the $\alpha-1$ chain, since in this case the Gly272Asp mutant displays a decrease of both the substrate affinity and the catalytic rate constant, suggesting that here the mutation affects also the substrate recognition mechanism (likely because of the presence of a negative charge).

We have previously demonstrated that MMP-1 uses an induced-fit mechanism to cleave collagen I [33]. As a whole, these data suggest that in the Gly272Asp mutant the interaction between the side chain of Asp and an amino acid in the catalytic domain makes the active site of MMP1 rigid, preventing the enzyme from efficiently cleaving the fibrillar collagen (Table 1). Thus, the substitution of Asp for Gly272 decreased the $k_{\text {cat }}$ and $K_{\mathrm{m}}$ values for the $\alpha-1$ chain by ten times, whereas for the $\alpha-2$ chain it lowered the affinity and catalytic activity of MMP-1 by 100 times and two times, respectively (see Table 1), leading to an overall similar $k_{\text {cat }} / K_{\mathrm{m}}$ value for both collagen chains. This is consistent with the lack of collagenolytic activity of MMPs with charged residues at this position of the hinge region, as occurs in stromelysin [51].

Together, these data indicate that the linker region (and in particular Gly272, which is located halfway along this region; see $[29,52])$ regulates the substrate-linked conformational change, which brings the catalytic site into close contact with the substrate after the binding of collagen I by the hemopexin-like domain. The substitution of the negatively charged Asp for Gly272 made the hinge region and the active site rigid, dramatically lowering the cleavage rate. The possible interaction of the Asp residue at this position of the hinge region with an amino acid(s) residue in the catalytic domain enhances the energy barrier for the bending transition, rendering the conformational change in the hinge region and the active site much more difficult, and rendering the cleavage event much more unlikely. This is underlined by a significant decrease in the affinity and catalytic activity of the enzyme for both collagen chains, even though the effect appears more pronounced for the enzymatic processing of the $\alpha-2$ chain than for the $\alpha-1$ chain. This is a clear indication that MMP-1 interacts differently with the two chains of collagen I (as also implied by the tenfold higher affinity for the $\alpha-1$ chain; see Table 1), involving different residues, such that the substitution of the negatively charged Asp for Gly272 does not affect the interaction with the $\alpha-2$ chain but decreases the affinity for the $\alpha-1$ chain. This difference for the interaction with the two chains is not unexpected, in view of the slightly different cleavage sites for the two chains $[44,50]$, but it seems to be eliminated by the replacement with Asp (but not with Ala or Asn; see Table 1), as if the discriminating recognition mechanism for the two chains lies in the presence of an uncharged residue at position 272 .

In conclusion, a concerted mechanism can be envisioned for the processing of the fibrillar collagen by MMP-1 (see Fig. 5). The overall process can be described as an intramolecular allosteric phenomenon, where a ligand-linked conformational change, involving the hinge domain as well as the catalytic domain, is triggered by the interaction of the hemopexin-like domain with either of the two chains of collagen I. This being the mechanism, wild-type MMP-1 seems to interact preferentially with the $\alpha-1$ chain, even though the cleavage catalytic rate-limiting step is faster for the $\alpha-2$ chain. This mechanism, which is likely operative also in other collagenases [32], opens up a new scenario for the design of more specific inhibitors for the activity of collagenases, suggesting that the primary requirement for a powerful inhibitory action could be the impairment of the 
hinge region flexibility in order to abolish the communication pathway between the hemopexin-like domain and the catalytic domain.

Acknowledgments The authors are grateful to $\mathrm{H}$. Nagase for a generous gift of wild-type MMP-1 and David Nelson from the University of Tennessee Health Science Center (Memphis, TN, USA) for technical support. This work was financially supported by the Italian Space Agency (ASI 2005 OSMA to U.T. and M.C.) and the National Institutes of Health (grants AR-41843 and CA-107183 to T.P.).

\section{References}

1. Nagase H, Woessner JF (1999) J Biol Chem 274:21491-21494

2. Vu TH, Werb Z (2000) Genes Dev 14:2123-2133

3. Sternlicht MD, Werb Z (2001) Annu Rev Cell Dev Biol 17:463-516

4. Aureli L, Gioia M, Cerbara I, Monaco S, Fasciglione GF, Marini S, Ascenzi P, Topai A, Coletta M (2008) Curr Med Chem 15:2192-2222

5. Wysocki AB, Staiano-Coico L, Grinnell F (1993) J Invest Dermatol 101:64-68

6. Matrisian LM, Bowden GT, Krieg P, Furstenberger G, Briand JP, Leroy P, Breathnach R (1986) Proc Natl Acad Sci USA 83:9413-9417

7. Hirose T, Riefe RA, Smith GN Jr, Stevens RM, Mainardi CL, Hasty KA (1992) J Rheumatol 19:593-599

8. Bafetti LM, Young TN, Itoh Y, Stack MS (1998) J Biol Chem 273:143-149

9. Vu TH, Shipley JM, Bergers G, Berger JE, Helms JA, Hanahan D, Shapiro SD, Senior RM, Werb Z (1998) Cell 93:411-422

10. Opdenakker G, Nelissen I, van Damme J (2003) Lancet Neurol 2:747-756

11. Murphy G, Allan JA, Willenbrock F, Cockett MI, O'Connell JP, Docherty AJP (1992) J Biol Chem 267:9612-9618

12. Hirose T, Patterson C, Pourmotabbed T, Mainardi CL, Hasty KA (1993) Proc Natl Acad Sci USA 90:2569-2573

13. Gioia M, Fasciglione GF, Marini S, D'Alessio S, De Sanctis G, Diekmann O, Pieper M, Politi V, Tschesche H, Coletta M (2002) J Biol Chem 277:23123-23130

14. Steffensen B, Wallon UM, Overall CM (1995) J Biol Chem 270:11555-11566

15. Wilson CL, Matrisian LM (1996) Int J Biochem Cell Biol 28:123-136

16. Ohuchi I, Imai K, Fujii Y, Sato H, Seiki M, Okada Y (1997) J Biol Chem 272:2446-2451

17. Prockop DJ, Kivirikko KI (1995) Annu Rev Biochem 64:403-434

18. Brodsky B, Ramshaw JA (1997) Matrix Biol 15:545-554

19. Ottl J, Gabriel D, Murphy G, Knäuper V, Tominaga Y, Nagase H, Kröger M, Tschesche H, Bode W, Moroder L (2000) Chem Biol 7:119-132

20. Shah K, Ramshaw JAM, Kirkpatrick A, Shah C, Brodsky B (1996) Biochemistry 35:10262-10268

21. Cawston TE (1996) Pharmacol Ther 70:163-182

22. Shingleton WD, Hodges DJ, Brick P, Cawston TE (1996) Biochem Cell Biol 74:759-775

23. Pilcher BK, Dumin JA, Sudbeck BD, Krane SM, Welgus HG, Parks WC (1997) J Cell Biol 137:1445-1457
24. Zaho WG, Byrne MH, Boyce BF, Krane SM (1999) J Clin Invest 103:517-524

25. Orgel JP, Wess TJ, Miller A (2000) Structure 8:137-142

26. Bozec L, Horton M (2005) Biophys J 88:4223-4231

27. Orgel JPRO, Irving TC, Miller A, Wess TJ (2006) Proc Natl Acad Sci USA 103:9001-9005

28. Brandstetter H, Grams F, Glitz D, Lang A, Huber R, Bode W, Krell HW, Engh RA (2001) J Biol Chem 276:17405-17412

29. Iyer S, Visse R, Nagase H, Acharya KR (2006) J Mol Biol 362:78-88

30. Marini S, Fasciglione GF, De Sanctis G, D’Alessio S, Politi V, Coletta M (2000) J Biol Chem 275:18657-18663

31. Chung L, Dinakarpandian D, Yoshida N, Lauer-Fields JL, Fields GB, Visse R, Nagase H (2004) EMBO J 23:3020-3030

32. Gioia M, Monaco S, Fasciglione GF, Coletti A, Modesti A, Marini S, Coletta M (2007) J Mol Biol 368:1101-1113

33. O'Farrell TJ, Guo R, Hasegawa H, Pourmotabbed T (2006) Biochemistry 45:15411-15418

34. Rosenblum G, Van den Steen PE, Cohen SR, Grossmann JG, Frenkel J, Sertchook R, Slack N, Strange RW, Opdenakker G, Sagi I (2007) Structure 15:1227-1236

35. Bertini I, Fragai M, Luchinat C, Melikian M, Mylonas E, Sarti N, Svergun DI (2009) J Biol Chem 284:12821-12828

36. Knäuper V, Docherty AJP, Smith B, Tschesche H, Murphy G (1997) FEBS Lett 405:60-64

37. Tsukada H, Pourmotabbed T (2002) J Biol Chem 277:2737827384

38. Bertini I, Fragai M, Luchinat C, Melikian M, Toccafondi M, Lauer JL, Fields GB (2012) J Am Chem Soc 134:2100-2110

39. O'Farrell TJ, Pourmotabbed T (2000) J Biol Chem 275:2796427972

40. Chandrakasan G, Torchia DA, Piez KA (1976) J Biol Chem 251:6062-6067

41. Bradford M (1976) Anal Biochem 72:248-254

42. Laemmli UK (1970) Nature 227:680-683

43. McBride DJ Jr, Choe V, Shapiro JR, Brodsky B (1997) J Mol Biol 270:275-284

44. Lauer-Fields JL, Tuzinski KA, Shimokawa K, Nagase H, Fields GB (2000) J Biol Chem 275:13282-13290

45. Mallya SK, Mookhtiar KA, Gao Y, Brew K, Dioszegi M, Birkedal-Hansen H, van Wart HE (1990) Biochemistry 29:10628-10634

46. Ackerman MS, Bhate M, Shenoy N, Beck K, Ramshaw JAM, Brodsky B (1999) J Biol Chem 274:7668-7673

47. Persikov AV, Ramshaw JAM, Kirkpatrick A, Brodsky B (2000) Biochemistry 39:14960-14967

48. Perona JJ, Tsu CA, Craik CS, Fletterik RJ (1997) Biochemistry 36:5381-5392

49. Lauer-Fields JL, Juska D, Fields GB (2002) Biopolymers 66:19-32

50. Salsas-Escat R, Nerenberg PS, Stultz CM (2010) Biochemistry 49:4147-4158

51. Chung L, Shimokawa K, Dinakarpandian D, Grams F, Fields GB, Nagase H (2000) J Biol Chem 275:29610-29617

52. Jozic D, Bourenkov G, Lim NH, Visse R, Nagase H, Bode W, Maskos K (2005) J Biol Chem 280:9578-9585

53. De Souza SJ, Pereira HM, Jacchieri S, Brentani RR (1996) FASEB J 10:927-930 